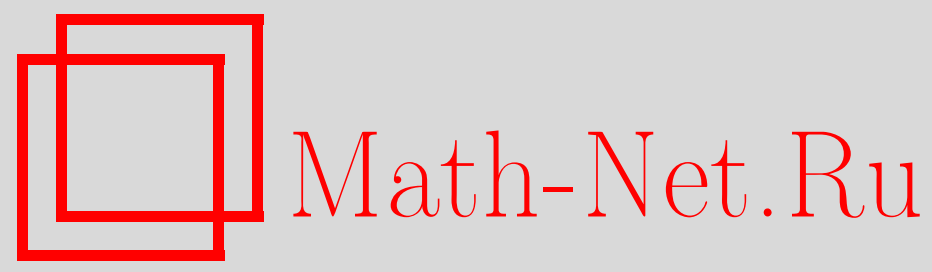

А. А. Туганбаев, Кольца Безу, многочлены и дистрибутивность, Матем. заметки, 2001, том 70, выпуск 2, 270-288

DOI: https://doi.org/10.4213/mzm740

Использование Общероссийского математического портала Math-Net.Ru подразумевает, что вы прочитали и согласны с пользовательским соглашением http://www.mathnet.ru/rus/agreement

Параметры загрузки:

IP: 54.84 .234 .179

26 апреля 2023 г., 13:31:44

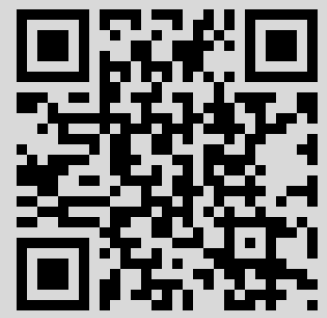




\title{
КОЛЬЦА БЕЗУ, МНОГОЧЛЕНЫ И ДИСТРИБУТИВНОСТЬ
}

\section{А. А. Туганбаев}

\begin{abstract}
Пусть $\varphi$-инъективный эндоморфизм кольца $A, A_{r}[x, \varphi] \equiv R$-правое кольцо косых многочленов. Если все правые аннуляторные идеалы кольца $A$ являются идеалами, то $R$-правое кольцо Безу $\Longleftrightarrow A$-риккартово справа правое кольцо Безу, $\varphi(e)=e$ для каждого центрального идемпотента $e \in A$ и элемент $\varphi(a)$ обратим в $A$ для каждого регулярного $a \in A$. Если $A$ строго регулярно и $n \geqslant 2$, то $R / x^{n} R$-правое кольцо Безу $\Longleftrightarrow R / x^{n} R$ - дистрибутивное справа кольцо $\Longleftrightarrow R / x^{n} R$ - инвариантное справа кольцо $\Longleftrightarrow \varphi(e)=e$ для каждого центрального идемпотента $e \in A$. Кольцо $R / x^{2} R$ дистрибутивно справа $\Longleftrightarrow R / x^{n} R$ дистрибутивно справа для всех натуральных $n$ $\Longleftrightarrow A$ дистрибутивно справа и риккартово справа или слева, $\varphi(e)=e$ для каждого центрального идемпотента $e \in A$ и $\varphi(a)$ обратимо в $A$ для каждого регулярного $a \in A$. Если кольцо $A$ является конечно-порожденным модулем над своим центром, то $A[x]-$ правое кольцо Безу $\Longleftrightarrow A[x] / x^{2} A[x]-$ правое кольцо Безу $\Longleftrightarrow A$-регулярное кольцо.

Библиограффия: 7 названий.
\end{abstract}

Введение. В настоящей работе все кольца предполагаются ассоциативными и с ненулевой единицей. Слова типа "кольцо Безу" означают, что соответствующие условия выполнены справа и слева. Если $A$ - кольцо и $\varphi$ - инъективный эндоморфизм кольца $A$, то через $A_{r}[x, \varphi]$ обозначается правое кольцо косых многочленов, состоящее из конечных формальных сумм $\sum x^{i} a_{i}$ от переменной $x$ с каноническими коэффициентами $a_{i} \in A$, где умножение определяется с помощью правила $a x^{i}=x^{i} \varphi^{i}(a)$. Модуль называется модулем Безу, если каждый его конечно-порожденньй подмодуль является циклическим модулем. Модуль называется дистрибутивным м, если решетка всех его подмодулей дистрибутивна. Через $r(X)$ обозначается правьй аннулятор подмножества $X$ правого модуля $M$. Правый модуль $M_{A}$ назьвается $р$ иккартовым, если для каждого $m \in M$ правый идеал $r(m)$ порождается идемпотентом кольца $A$. Элемент кольца $A$ назьвается регулярным справа (слева), если он не является левым (правым) делителем нуля в $A$. Кольцо называется инвариантным справа, если каждьй его правый идеал является идеалом. Кольцо $A$ назьвается строго регулярным, если для каждого $a \in A$ существует такое $b \in A$, что $a=a^{2} b$ (это равносильно тому, что каждьй главный правьй идеал кольца $A$ порождается центральным идемпотентом; см., например, $[1,3.30])$.

Известно, что кольцо многочленов $A[x]$ над строго регулярным кольцом $A$ является кольцом Безу (см., например, $[1,12.3])$. В [2, предложение 8] доказано, что если $A$ - строго регулярное кольцо, то $A[x] / x^{n} A[x]$ - инвариантное кольцо для любого натурального числа $n$. Основными результатами работы являются теоремы 1-4.

Работа выполнена при поддержке Российского фонда фундаментальных исследований, грант № 99-01-00382. 


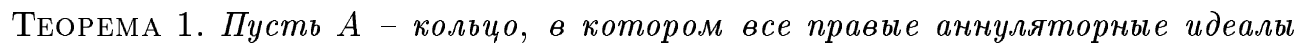
являются идеалами, $\varphi$ - инвективный эндоморфизм кольиа А. Тогда равносильны следующие условия:

(1) $A_{r}[x, \varphi]-$ правое кольио Безу;

(2) $A_{r}[x, \varphi] / x^{2} A_{r}[x, \varphi]-$ правое кольцо Безу;

(3) $A_{r}[x, \varphi]$ - редуцированное правое кольцо Безу;

(4) $A$ - риккартово справа правое кольио Безу, $\varphi(e)=е$ для каждого иентрального идемпотента $е \in A$ и әлемент $\varphi(d)$ обратим в $A$ для каждого регулярного әлемента $d \in A$.

Теорема 2. Пусть $A$ - строго регулярное кольио, $\varphi-$ ингективный әндоморфизм кольца $A$ и $n$ - натуральное число $\geqslant 2$. Тогда равносильны следующие условия:

(1) $A_{r}[x, \varphi] / x^{n} A_{r}[x, \varphi]$ - инвариантное справа кольцо;

(2) $A_{r}[x, \varphi] / x^{n} A_{r}[x, \varphi]$ - нормальное кольцо;

(3) $A_{r}[x, \varphi] / x^{n} A_{r}[x, \varphi]-$ правое кольцо Безу;

(4) $A_{r}[x, \varphi] / x^{n} A_{r}[x, \varphi]$ - дистрибутивное справа кольио;

(5) $A_{r}[x, \varphi]$ - редуиированное правое кольцо Безу и $A_{r}[x, \varphi] / x^{k} A_{r}[x, \varphi]-$ инвариантное справа дистрибутивное справа правое кольцо Безу для каждого натурального числа $k$;

(6) $\varphi(e)=е$ для каждого иентрального идемпотента е $\in A$.

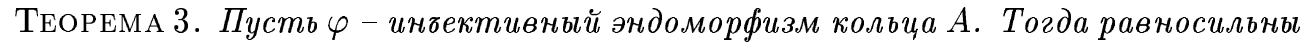
следуюшие условия:

(1) $A_{r}[x, \varphi] / x^{2} A_{r}[x, \varphi]-$-истрибутивное справа кольцо;

(2) $A_{r}[x, \varphi] / x^{n} A_{r}[x, \varphi]$ - дистрибутивное справа кольцо для всех натуральных чисел $n$;

(3) A - дистрибутивное справа риккартово справа кольцо, $\varphi(e)=е$ для каждого центрального идемпотента $е \in A$ и әлемент $\varphi(d)$ обратим в $A$ для каждого регулярного әлемента $d \in A$.

ТЕОРема 4. Пусть $A-$ кольчо, являюшееся конечно-порохсденным модулем над своим центром. Тогда равносильны следующие условия:

(1) $A[x]-$ правое кольцо Безу;

(2) $A[x] / x^{2} A[x]-$ правое кольио Безу;

(3) $A-$ регулярное кольио.

ЗАмЕчАнИЕ 1. В связи с приведенньми вьше теоремами заметим, что существует коммутативная цепная область $A$, не являющаяся строго регулярным кольцом и обладающая инъективным эндоморфизмом $\varphi$ таким, что элемент $\varphi(a)$ обратим в $A$ для любого ненулевого $a \in A$. (В частности, $A$ - коммутативная дистрибутивная область Безу и $\varphi(J(A) \backslash 0) \subseteq A \backslash J(A)$.) Пусть $k$ - поле, $F$ - поле рациональных функций над $k$ от счетного числа переменных $\left\{t_{i}\right\}_{i=0}^{\infty}$. Положим $t \equiv t_{0}$. Существует инъективньй эндоморфизм $\alpha$ поля $F$ такой, что $\alpha\left(t_{i}\right)=t_{i+1}$ для всех $i$; элемент $t$ трансцендентен над $\alpha(F)$. Пусть $A$ - кольцо, образованное всеми дробями $f /(1+y g)$, где $f, g$-произвольные многочлены из кольца многочленов $F[y]$. С помощью правила $\varphi(y)=t$ можно продолжить $\alpha$ до инъективного эндоморфизма $\varphi$ кольца $A$. Непосредственно проверяется, что $A-$ 
коммутативная цепная область главных идеалов, не являющаяся полем, причем элемент $\varphi(a)$ обратим в $A$ для любого ненулевого $a \in A$. Так как область $A$ не является полем, то область $A$ не является строго регулярным кольцом.

Доказательства теорем 1-4 разбиты на ряд лемм. Приведем необходимые определения и обозначения. Модуль называется иепным, если любые два его подмодуля сравнимы по включению. Модуль $M$ называется конечномерным, если $M$ не содержит бесконечных прямых сумм ненулевых подмодулей. Через $J(A)$ обозначается радикал Джекобсона кольца $A$. Кольцо $A$ назьвается ортогонально конечным, если $A$ не содержит бесконечных множеств ненулевых ортогональных идемпотентов. Кольцо назьвается регулярным, если любой его главньй правьй идеал порождается идемпотентом. Кольцо $A$ назьвается бирегулярным, если любой его 1-порожденньй двусторонний идеал порождается центральным идемпотентом. Кольцо без ненулевых нильпотентных элементов называется редуиированным кольцом. Кольцо назьвается нормальным, если все его идемпотенты центральны. Кольцо называется прямо-конечным если все его обратимые справа или слева элементы обратимы. Кольцо назьвается квази-инвариантным справа, если все его максимальные правые идеалы являются идеалами. Конечномерное справа кольцо с условием максимальности для правых аннуляторов называется правым кольиом Голди. Факторкольцо $A / P$ называется пирсовским слоем кольца $A$, если идеал $P$ является максимальным (по включению) элементом непустого множества всех собственных идеалов кольца $A$, порожденных центральными идемпотентами.

\section{1. Доказательство теоремы 1.}

Лемма 1.1. (1) Если $A$ - редуиированное кольцо, то $A$ - нормальное кольцо и $r(X)=\ell(X)$ - идеал в $A$ для любого подмнохсества $X \subseteq A$.

(2) Если $A$ - риккартово справа нормальное кольцо, то $A$ - риккартово слева редуиированное кольио, в котором каждый әлемент является произведением центрального идемпотента на регулярный әлемент.

(3) Если d-регулярный әлемент кольца $A$ и $A / P$ - пирсовский слой кольиа $A$, то $d+P-$ регулярный әлемент кольиа $A / P$.

(4) Если $A$ - нормальное кольцо, то А прямо-конечно и каждый его пирсовский слой не имеет нетривиальных идемпотентов.

(5) Если $A$ - риккартово справа нормальное кольцо, то каждый его пирсовский слой $A / P$ является областью.

(6) Допустим, что существует такой инвективный эндоморфизм $\varphi$ кольиа $A$, ито $а \in$ ач $(a) A$ для любого $a \in A$. Тогда $\varphi(e)=е$ для любого иентрального идемпотента $е \in A$ и для кажсдого регулярного справа әлемента $d \in A$ әлемент $\varphi(d)$ обратим справа в кольие $A$.

ДокАЗАТЕльство. (1), (2) и (3) доказаны в $[3,1.35(1), 3.36,6.89(\mathrm{ii})]$.

(4) Так как $A$ нормально, то каждьй его пирсовский слой не имеет нетривиальных идемпотентов [3, 6.91]. Пусть $a, b \in A$ и $a b=1$. Тогда $(b a)^{2}=b a$. По условию $b a-$ центральньй идемпотент. Поэтому $b a a=a b a=a$. Тогда $b a=b a a b=a b=1$.

(5) Пусть $a, b \in A \backslash P$. Согласно (2) сушествуют регулярные элементы $c, d \in A$ и центральные идемпотенты $e, f \in A$ такие, что $a=c e$ и $b=d f$. Тогда $e, f \in A \backslash P$. Кроме того, факторкольцо $A / P$ не имеет нетривиальных идемпотентов согласно (4). Поэтому 
$1-e, 1-f \in P$. Тогда $a+P=c+P$ и $b+P=d+P$. Согласно $(3) a+P$ и $b+P-$ регулярные элементы факторкольца $A / P$. Поэтому $A / P$ - область.

(6) По условию существуют $b, c \in A$ такие, что $e=e \varphi(e) b$ и $1-e=(1-e)(1-\varphi(e)) c=$ $(1-\varphi(e)) c(1-e)$. Тогда

$$
e=e \varphi(e) b=e(\varphi(e))^{2} b=\varphi(e) e \varphi(e) b=\varphi(e) e=e \varphi(e)
$$

и

$$
\begin{aligned}
\varphi(e) & =(e+(1-e)) \varphi(e)=e \varphi(e)+(1-e) \varphi(e) \\
& =e+\varphi(e)(1-e)=e+\varphi(e)(1-\varphi(e)) c(1-e)=e .
\end{aligned}
$$

По условию существует элемент $m \in A$ такой, что $(1-\varphi(d) m) \in r(d)=0$. Поэтому элемент $\varphi(d)$ обратим справа.

Лемма 1.2. Пусть $A-$ кольио, в котором все правые аннуляторы являются

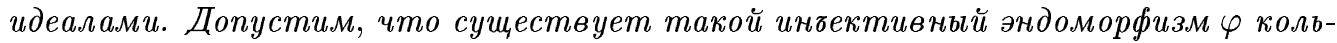
иа $A$, ито $а \in \operatorname{a\varphi }($ a) $A$ для любого $а \in A$.

(1) $A$ нормально, $r(a)=r(\varphi(a))$ для любого $a \in A$ и $\varphi(e)=е$ для любого идемпотента е $\in A$.

(2) $A$ - редуцированное риккартово кольцо и для любого а $\in A$ существует $b \in A$ такое, что $\varphi(a) b$ и $1-\varphi(a) b$ - иентральные идемпотенты, $a=a \varphi(a) b$, $\varphi(a)=\varphi(a) b \varphi(a)=(\varphi(a))^{2} b$ ur $(a)=\ell(a)=(1-\varphi(a) b) A$.

(3) Для любого регулярного справа әлемента $d \in A$ әлемент $\varphi(d)$ обратим в кольие $A$.

(4) Пусть $A / P$ - пирсовский слой кольиа $A$. Тогда $\varphi(P) \subseteq P, A / P$ - область, $\varphi$ индуиирует инбективный әндоморфизм $\bar{\varphi}$ области $A / P$ и $\bar{\varphi}(a+P)$ - обратимый әлемент области $A / P$ для кажсдого ненулевого әлемента $a+P \in A / P$.

ДоказАтЕльство. Так как в кольце $A$ все правые аннуляторы являются идеалами, то кольцо $A$ нормально.

(1) По лемме 1.1(6) $\varphi(e)=e$ для любого идемпотента $e \in A$. Пусть $a \in A$. По условию существует элемент $b \in A$ такой, что $a=a \varphi(a) b$. Пусть $x \in r(\varphi(a))$. Так как $r(\varphi(a))$ - идеал, то $b x \in r(\varphi(a))$. Поэтому $\varphi(a) b x=0$, откуда $a x=a \varphi(a) b x=0$. Тогда $r(\varphi(a)) \subseteq r(a)$.

Пусть $y \in r(a)$. Тогда $\varphi(y) \in r(\varphi(a))$. По условию существует элемент $z \in A$ такой, что $y=y \varphi(y) z$. Так как $r(\varphi(a))$ - идеал и $\varphi(y) \in r(\varphi(a))$, то верно включение $y \varphi(y) \in \varphi(a)$. Поэтому $\varphi(a) y \varphi(y)=0$. Тогда $\varphi(a) y=\varphi(a) y \varphi(y) z=0$. Поэтому $r(a) \subseteq r(\varphi(a))$.

(2) Пусть $x \in A$ и $x^{2}=0$. Согласно (1) $r(x)=r(\varphi(x))$. Так как $x \in r(x)$, то верно равенство $x \varphi(x)=0$. По условию $x \in x \varphi(x) A$. Поэтому $x=0$ и $A$ - редуцированное кольцо.

Пусть $a \in A$. По условию существует элемент $b \in A$ такой, что $a=a \varphi(a) b$. Тогда $1-\varphi(a) b \in r(a)$. Из (1) и леммы 1.1(1) следует, что $r(a)=\ell(a)=r(\varphi(a))=\ell(\varphi(a))$. Поэтому $0=\varphi(a)(1-\varphi(a) b)=(1-\varphi(a) b) \varphi(a)$. Тогда $\varphi(a)=\varphi(a) b \varphi(a)=(\varphi(a))^{2} b$. Поэтому $\varphi(a) b$ и $1-\varphi(a) b$-идемпотенты. Согласно $(1) \varphi(a) b$ и $1-\varphi(a) b$-центральные идемпотенты. Так как $1-\varphi(a) b \in r(a)$, то остается доказать включение $r(a) \subseteq(1-\varphi(a) b) A$. 
Если $y \in r(a)=\ell(\varphi(a))$, то $y=y(1-\varphi(a) b) \in A(1-\varphi(a) b)=(1-\varphi(a) b) A$. Поэтому идеал $r(a)$ порождается центральным идемпотентом $1-\varphi(a) b$ и $A$ - риккартово кольцо.

(3) По лемме 1.1(6) элемент $\varphi(d)$ обратим справа. По лемме 1.1(4) $\varphi(d)$ - обратимый элемент.

(4) По лемме 1.1(5) $A / P$ - область. Пусть $h: A \rightarrow A / P$ - естественный эпиморфизм и $P=\sum_{i \in I} e_{i} A$, где все $e_{i}$ - центральные идемпотенты. Согласно $(1) \varphi\left(e_{i}\right)=e_{i}$ для всех $e_{i}$. Поэтому $\varphi(P) \subseteq P$ и $\varphi$ индуцирует эндоморфизм $\bar{\varphi}$ области $A / P$. Пусть $a \in A \backslash P$. По лемме 1.1(2) $a=e d$, где $e$ - центральньй идемпотент и $d$ - регулярный элемент. Так как $a=e d \in A \backslash P$, то верно включение $e \in A \backslash P$. Согласно (3) $\varphi(d)$ - обратимый элемент в $A$. Поэтому $h(\varphi(d))$ - обратимый элемент в $A / P$. Coгласно (1) $\varphi(e)=e \in A \backslash P$ и $h(\varphi(e))=h(e)=h(1)$ - единица кольца $A / P$. Поэтому $e=\varphi(e)=\varphi(e) \varphi(d)(\varphi(d))^{-1}=\varphi(a) \varphi(d)^{-1} \in A \backslash P$. Тогда $\varphi(a) \in A \backslash P$ и $h(1)=h(\varphi(a)) h\left(\varphi(d)^{-1}\right)$. Поэтому $\operatorname{Ker}(\bar{\varphi})=0$ и $h(\varphi(a))=\bar{\varphi}(a+P)$ - обратимьй элемент области $A / P$.

Лемма 1.3. Пусть $\varphi$-инбективный әндоморфизм кольиа $A, R \equiv A_{r}[x, \varphi]$. Допустим, что $R / x^{2} R$ - правое кольио Безу.

(1) $A$ - правое кольчо Безу и для каждого а $\in A$ существуют $f_{0}, g_{0}, u_{0}, v_{0}, b, c \in A$ такие, что выполняются следующие равенства:

(i) $a=a f_{0} u_{0}$;

(ii) $a f_{0} v_{0}=0$;

(iii) $1=\varphi(a) b+g_{0} v_{0}$;

(iv) $\varphi(a) c=g_{0} u_{0}$.

(2) Если в кольце $A$ все правые аннуляторы являются идеалами, то для любого $a \in A$ существует $b \in A$ mакое, что $a=a \varphi(a) b$.

ДокАЗАТЕЛЬСтво. (1) Так как существует сюръективньй гомоморфизм $R / x^{2} R \rightarrow$ $R / x R \cong A$, то $A$ - правое кольцо Безу. Пусть $h: R \rightarrow R / x^{2} R$ - естественный эпиморфизм. Так как $h(R)$ - правое кольцо Безу, то $h(a R+x R)$ - главный правый идеал кольца $h(R)$. Поэтому существуют многочлены $f, g \in R$ такие, что $h(a R+x R)=$ $h((a f+x g) R)$. Тогда сушествуют многочлены $u, v \in R$ такие, что $(a f+x g) u-a \in x^{2} R$ и $(a f+x g) v-x \in x^{2} R$. Пусть $f_{0}, g_{0}, u_{0}$ и $v_{0}$ - свободные члены многочленов $f, g, u, v$ соответственно, $f_{1}, u_{1}, v_{1}$ - канонические коэффициенты при $x$ многочленов $f, u, v$ соответственно. Так как свободные члены и коэффициенты при $x$ многочленов $(a f+x g) u-a$ и $(a f+x g) v-x$ равны нулю, то получаем следующие равенства:

$$
\begin{gathered}
a f_{0} u_{0}-a=0, \quad a f_{0} v_{0}=0, \\
\varphi(a)\left(\varphi\left(f_{0}\right) u_{1}+f_{1} u_{0}\right)+g_{0} u_{0}=0, \quad \varphi(a)\left(\varphi\left(f_{0}\right) v_{1}+f_{1} v_{0}\right)+g_{0} v_{0}-1=0 .
\end{gathered}
$$

Теперь можно положить $b \equiv \varphi\left(f_{0}\right) v_{1}+f_{1} v_{0}, c \equiv-\varphi\left(f_{0}\right) u_{1}-f_{1} u_{0}$.

(2) Согласно (1) существуют $f_{0}, g_{0}, u_{0}, v_{0}, b \in A$ такие, что верны следующие равенства:

(i) $a=a f_{0} u_{0}$;

(ii) $a f_{0} v_{0}=0$;

(iii) $1=\varphi(a) b+g_{0} v_{0}$. 
Согласно (ii) $v_{0} \in r\left(a f_{0}\right)$. Так как $r\left(a f_{0}\right)$ - идеал в $A$ по условию, то верно включение $u_{0} g_{0} v_{0} \in r\left(a f_{0}\right)$. Поэтому $a f_{0} u_{0} g_{0} v_{0}=0$. Из $(\mathrm{i})$ следует, что $a\left(g_{0} v_{0}\right)=\left(a f_{0} u_{0}\right) g_{0} v_{0}=0$. Из (iii) следует, что $a=a \cdot 1=a \varphi(a) b+a g_{0} v_{0}$. Так как $a g_{0} v_{0}=0$, то верно равенство $a=a \varphi(a) b$.

Лемма 1.4. Пусть $A-$ область, $\varphi$ - инвективный эндоморфизм области $A$ такой, что әлемент $\varphi(a)$ обратим в $A$ для каждого ненулевого $а \in A$. Положим $R \equiv A_{r}[x, \varphi]$.

(1) Для любых двух таких ненулевых многочленов $f, g \in R$, ито $\operatorname{deg}(f)<\operatorname{deg}(g)$, существует многочлен $h \in R$ такой, что $\operatorname{deg}(h)<\operatorname{deg}(g)$ u $f R+g R=f R+h R$.

(2) Если $G$ - ненулевой правый идеал кольиа $R$ u $m \equiv \min _{0 \neq g \in G} \operatorname{deg}(g)$, то правый идеал $G$ порождается многочленами степени $\mathrm{m}$.

(3) Если $F$ - конечно-порожденный ненулевой правый идеал кольца $R u$

$$
m \equiv \min _{0 \neq f \in F} \operatorname{deg}(f)
$$

то правый идеал $F$ порожден конечным числом многочленов степени $\mathrm{m}$.

(4) Для любого ненулевого многочлена $f \in R$ существуют иелое число $m \geqslant 0$, ненулевой әлемент $f_{0} \in A$ и многочлен $s \in R$ такие, что $f R=x^{m} f_{0}(1+x s) R$.

(5) Пусть $F$ и $G$ - главные правые идеаль кольиа $R, n$ - натуральное число, $h: R \rightarrow R / x^{n} R$ - естественный әпиморфизм. Допустим, что главные правые идеаль $h(F)$ и $h(G)$ кольиа $h(R)$ не сравнимы по включению. Тогда существуют не сравнимые по включению главные правые идеалы $F_{0}, G_{0}$ кольца $A$ и иелое число $m \geqslant 0$ такие, что $h(F)=h\left(x^{m}\right) h\left(F_{0}\right) h(R), h(G)=h\left(x^{m}\right) h\left(G_{0}\right) h(R)$, $h(F) \cap h(G)=h\left(x^{m}\right) h\left(F_{0} \cap G_{0}\right) h(R), h(F)+h(G)=h\left(x^{m}\right) h\left(F_{0}+G_{0}\right) h(R)$.

ДокАЗАтЕЛЬСтво. (1) Положим $m \equiv \operatorname{deg}(f) \geqslant 0$ и $\operatorname{deg}(g) \equiv n>m$. Тогда существуют ненулевые $a, b \in A$ такие, что $f=x^{m} a+s$ и $g=x^{n} b+t$, где $\operatorname{deg}(s)<m$ и $\operatorname{deg}(t)<n$. Обратимьй справа элемент $\varphi(a)$ области $A$ обратим в $A$. Тогда $x^{n-m}=$ $a x^{n-m}\left(\varphi^{n-m}(a)\right)^{-1}$. Поэтому

$$
g-t=x^{n} b=x^{m} a x^{n-m} \varphi^{n-m}(a)^{-1} b=(f-s) x^{n-m} \varphi^{n-m}(a)^{-1} b .
$$

Положим

$$
h \equiv g-f x^{n-m} \varphi^{n-m}(a)^{-1} b=t-s x^{n-m} \varphi^{n-m}(a)^{-1} b \in f R+g R .
$$

Тогда $\operatorname{deg}(h)<n$ и $g=f x^{n-m} \varphi^{n-m}(a)^{-1} b+h \in f R+h R$. Поэтому

$$
f R+g R \subseteq f R+h R \subseteq f R+g R
$$

(2) Пусть $F$ - ненулевой правьй идеал кольца $R$, порожденньй всеми многочленами степени $m$, лежащими в $G$. Допустим, что $F \neq G$. Тогда существует целое число $n>m$ такое, что все многочлены из $G$ степени $<n$ лежат в $F$ и $\operatorname{deg}(g)=n$ для некоторого многочлена $g \in G \backslash F$. В правом идеале $F$ возьмем произвольньй ненулевой многочлен $f$ степени $m$. Согласно (1) существует многочлен $h \in R$ такой, что $\operatorname{deg}(h)<n$ и $f R+g R=f R+h R$. Так как $h \in G$ и $\operatorname{deg}(h)<n$, то $h \in F$. Поэтому $g \in f R+h R \subseteq F$. Получено противоречие. 
(3) следует из (2) и того, что из каждой системы образующих конечно-порожденного правого идеала можно выбрать конечную систему образующих.

(4) Для $f$ существуют целое число $m \geqslant 0$, ненулевой элемент $f_{0} \in A$ и многочлен $s \in R$ такие, что

$$
f=x^{m}\left(f_{0}+x s\right)=x^{m} f_{0}\left(1+x\left(\varphi\left(f_{0}\right)\right)^{-1} s\right) .
$$

Поэтому $f R=x^{m} f_{0}(1+x s) R$.

(5) Согласно (4) существуют целые числа $k \geqslant 0$ и $m \geqslant 0$, ненулевые элементы $f_{0}, g_{0} \in A$ и многочлены $s, t \in R$ такие, что $F=x^{k} f_{0}(1+x s) R$ и $G=x^{m} g_{0}(1+x t) R$. Так как $h(x s)$ и $h(x t)$ - нильпотентные элементы кольца $h(R)$, то верны равенства

$$
h(R)=h(1+x s) h(R)=h(1+x t) h(R) .
$$

Поэтому $h(F)=h\left(x^{k}\right) h\left(f_{0}\right) h(R)$ и $h(G)=h\left(x^{m}\right) h\left(g_{0}\right) h(R)$. Допустим, что $k \neq m$. Например, пусть $k<m$. Тогда

$$
x^{m} g_{0}=x^{k} x^{m-k} g_{0}=x^{k} f_{0} x^{m-k}\left(\varphi^{m-k}\left(f_{0}\right)\right)^{-1} g_{0} \in x^{k} f_{0} R .
$$

Поэтому $h(G)=h\left(x^{m} g_{0}\right) h(R) \subseteq h\left(x^{k} f_{0}\right) h(R)=h(F)$. Тогда главные правые идеалы $h(F)$ и $h(G)$ кольца $h(R)$ сравнимы по включению. Получено противоречие. Следовательно, $k=m$. Тогда $h(F)=h\left(x^{m}\right) h\left(F_{0}\right) h(R)$ и $h(G)=h\left(x^{m}\right) h\left(G_{0}\right) h(R)$, где $F_{0} \equiv f_{0} A$ и $G_{0} \equiv g_{0} A$. Так как $h(F)$ и $h(G)$ не сравнимы по включению, то $F_{0}$ и $G_{0}$ не сравнимы по включению. Равенства

$$
h(F) \cap h(G)=h\left(x^{m}\right) h\left(F_{0} \cap G_{0}\right) h(R) \quad \text { и } \quad h(F)+h(G)=h\left(x^{m}\right) h\left(F_{0}+G_{0}\right) h(R)
$$

проверяются непосредственно.

ЛЕмма 1.5. Пусть $A-$ область и $\varphi$-ее инвективный әндоморфизм. Равносильны следующие условия:

(1) $A_{r}[x, \varphi]-$ правое кольцо Безу;

(2) $A_{r}[x, \varphi] / x^{2} A_{r}[x, \varphi]-$ правое кольцо Безу;

(3) $A$ - правое кольцо Безу и $\varphi(a) A=A$ для каждого ненулевого $a \in A$.

ДокАЗАТЕЛЬСтво. Положим $R \equiv A_{r}[x, \varphi]$. Импликация $(1) \Longrightarrow(2)$ очевидна.

$(2) \Longrightarrow(3)$. По лемме $1.3 A$ - правая область Безу и $a \in a \varphi(a) A$ для любого $a \in A$. По лемме $1.2(3) \varphi(a) A=A$ для каждого ненулевого $a \in A$.

$(3) \Longrightarrow(1)$. Пусть $F$ - конечно-порожденньй ненулевой правьй идеал кольца $R$ и $m \equiv \min _{0 \neq f \in F} \operatorname{deg}(f)$. По лемме $1.4(3)$ существуют ненулевые многочлены $f_{1}, \ldots, f_{n}$ степени $m \geqslant 0$ такие, что $F=\sum_{i=1}^{n} f_{i} R$. Пусть $a_{i}$ - ненулевые старшие коэффициенты многочленов $f_{i}(i=1, \ldots, n)$. Так как $A$ - правое кольцо Безу, то существуют $b_{1}, \ldots, b_{n}, c_{1}, \ldots, c_{n} \in A$ такие, что

$$
\left(\sum_{i=1}^{n} a_{i} b_{i}\right) c_{j}=a_{j}, \quad j=1, \ldots, n .
$$

Положим $g \equiv \sum_{i=1}^{n} f_{i} b_{i} \in F, h_{j} \equiv f_{j}-g c_{j} \in F$. Тогда $\operatorname{deg}\left(h_{j}\right)<m$. Так как $m \equiv \min _{0 \neq f \in F} \operatorname{deg}(f)$, тополучаем равенства $h_{j}=0$ и $f_{j}=g c_{j}(j=1, \ldots, n)$. Поэтому $F=g R$. 
ЛЕмма $1.6[3,6.90]$. Пусть $a_{1}, \ldots, a_{m}$ - әлементы кольиа $A$, и пусть $f_{1}, \ldots, f_{k}-$ многочлены с иелыми коэффичиентами от некоммутирующих переменных $x_{1}, \ldots$, $x_{m}, y_{1}, \ldots, y_{n}$. Равносильны следуюшие условия:

(1) существуют әлементы $b_{1}, \ldots, b_{n} \in A$ такие, что

$$
f_{i}\left(a_{1}, \ldots, a_{m}, b_{1}, \ldots, b_{n}\right)=0 \quad \text { npu } i=1, \ldots, k
$$

(2) для произвольного пирсовского слоя $\bar{A}$ кольца $A$ существуют әлементы $\bar{b}_{1}, \ldots, \bar{b}_{n} \in \bar{A}$ maкue, чmo

$$
f_{i}\left(h\left(a_{1}\right), \ldots, h\left(a_{m}\right), \bar{b}_{1}, \ldots, \bar{b}_{n}\right)=0 \quad \text { npu } i=1, \ldots, k,
$$

әде $h: A \rightarrow \bar{A}-$ естественныц эпиморфизм.

Лемма 1.7. $A$ - правое кольцо Безу является правым кольцом Безу.

ДокАЗАТЕЛЬСТво. Импликация $\Longrightarrow$ следует из того, что каждое факторкольцо правого кольца Безу является правым кольцом Безу.

$\Longleftarrow$ Пусть $a_{1}, a_{2} \in A$ и $x_{1}, x_{2}, y_{1} y_{2}, y_{3}, y_{4}$ - некоммутирующие переменные. Обозначим через $f_{1}\left(x_{1}, x_{2}, y_{1} y_{2}, y_{3}, y_{4}\right)$ и $f_{2}\left(x_{1}, x_{2}, y_{1} y_{2}, y_{3}, y_{4}\right)$ многочлены $\left(x_{1} y_{1}+x_{2} y_{2}\right) y_{3}-x_{1}$ и $\left(x_{1} y_{1}+x_{2} y_{2}\right) y_{4}-x_{2}$ соответственно. Пусть $\bar{A}$ - произвольный пирсовский слой кольца $A$ и $h: A \rightarrow \bar{A}$ - естественный эпиморфизм. По условию $\bar{A}$ - правое кольцо Безу. Поэтому существуют $\bar{b}_{1}, \bar{b}_{2}, \bar{b}_{3}, \bar{b}_{4} \in \bar{A}$ такие, что $f_{i}\left(h\left(a_{1}\right), h\left(a_{2}\right), \bar{b}_{1}, \bar{b}_{2}, \bar{b}_{3}, \bar{b}_{4}\right)=0$ при $i=1,2$. По лемме 1.6 существуют $b_{1}, b_{2}, b_{3}, b_{4} \in A$ такие, что $f_{i}\left(a_{1}, a_{2}, b_{1}, b_{2}, b_{3}, b_{4}\right)=0$ при $i=1,2$. Поэтому $A$ - правое кольцо Безу.

ЛЕмма 1.8. Пусть $\varphi-$ инбективный эндоморфизм кольиа $A, R \equiv A_{r}[x, \varphi], n-$ натуральное число $\geqslant 2 u h: R \rightarrow R / x^{n} R$ - естественный эпиморфизм.

(1) Если $f$-многочлен из $R$ такой, что $h(f)$ - ненулевой идемпотент кольиа $h(R)$, то свободный член $f_{0}$ многочлена $f$ является ненулевым идемпотентом.

(2) Если $f$-многочлен из $R$ такой, что $h(f)$ лежит в иентре кольиа $h(R)$, то $f_{0}$ лежит в иентре кольца $R$ и $f_{0}=\varphi\left(f_{0}\right)$.

(3) Eсли $f$ - многочлен из $R$ такой, что $h(f)$ - идемпотент кольца $h(R)$ и $f_{0}$ иентральный идемпотент кольца $R$, то $h(f)=h\left(f_{0}\right)$.

(4) Eсли $f$ - такой многочлен из $R$, что $h(f)$ - чентральный идемпотент кольиа $h(R)$, то $f_{0}$ - центральный идемпотент кольиа $R, f_{0}=\varphi\left(f_{0}\right) \in A u$ $h(f)=h\left(f_{0}\right)$.

(5) Если $f$ - иентральный идемпотент кольца $R$, то $f$ содерэится в кольие $A$ $u f=\varphi(f)$.

(6) Пусть $D(A)$ - мнохество всех таких центральных идемпотентов е кольиа $A$, что $\varphi(e)=е$. Тогда мнохсество всех иентральных идемпотентов кольиа $R$ совпадает с $D(A)$ и множество всех иентральных идемпотентов кольиа $h(R)$ совпадает с $h(D(A))$.

(7) Если кольцо $R$ конечномерно слева, то $\varphi(A) \varphi(A)$ - существенный подмодуль модуля $\varphi(A) A$.

(8) Если $A$ - полупростое артиново кольцо и $R$ конечномерно слева, то $\varphi-$ автоморфизм. 
ДоказАтельство. (1) Так как $h(f) \neq 0$, то $f \neq 0$. По условию $f-f^{2} \in x^{n} R$. Поэтому свободньй член $f_{0}-f_{0}{ }^{2}$ многочлена $f-f^{2}$ равен нулю и $f_{0}$ - идемпотент. Допустим, что $f_{0}=0$. Тогда существует $k \geqslant 1$ такое, что $f=x^{k}(a+x g)$, где $0 \neq a \in A$ и $g \in R$. Так как $h(f) \neq 0$, то $k<n$. Тогда $a-$ коэффициент при $x^{k}$ многочлена $f-f^{2} \in x^{n} R$. Поэтому $a=0$. Получено противоречие.

$(2)$ Коэффициент при $x$ многочлена $x f-f x$ равен $f_{0}-\varphi\left(f_{0}\right)$. Так как $x f-f x \in x^{n} R$ и $n \geqslant 2$, то верно равенство $f_{0}-\varphi\left(f_{0}\right)=0$. Поэтому $f_{0}=\varphi\left(f_{0}\right)$, откуда $f_{0} x=x f_{0}$. Пусть $a \in A$. Свободньй член многочлена $f a-a f$ равен $f_{0} a-a f_{0}$. Так как $f a-a f \in x^{n} R$, то верно равенство $f_{0} a=a f_{0}$. Кроме того, $f_{0} x=x f_{0}$. Поэтому $f_{0}$ лежит в центре кольца $R$.

(3) Допустим, что $h(f)=h\left(f f_{0}\right)$. Тогда существует многочлен $g \in R$ такой, что $h(f)=h(1+x g) h\left(f_{0}\right)$. Так как $h\left(f_{0}\right)$ и $h(f)$ - идемпотенты кольца $h(R)$ и $h\left(f_{0}\right)$ лежит в центре кольца $R$, то верно равенство $h(1+x g) h\left(f_{0}\right)=(h(1+x g))^{2} h\left(f_{0}\right)$. Элемент $h(1+x g)$ обратим в кольце $h(R)$, поскольку элемент $h(x g)$ нильпотентен. Поэтому $h\left(f_{0}\right)=h(1+x g) h\left(f_{0}\right)=h(f)$.

Допустим, что $h(f) \neq h\left(f f_{0}\right)$. Так как $h\left(f_{0}\right)$ - центральный идемпотент кольца $h(R)$, то $h\left(1-f_{0}\right)$ - центральньй идемпотент кольца $h(R)$. Кроме того, $h(f)$ - идемпотент. Тогда $h(f) h\left(1-f_{0}\right)$ - ненулевой идемпотент кольца $h(R)$. Кроме того, свободньй член многочлена $f\left(1-f_{0}\right)$ равен нулю. Это противоречит $(1)$.

(4) Согласно (1) и (2) $f_{0}$ - центральньй идемпотент кольца $R$ и $f_{0}=\varphi\left(f_{0}\right) \in A$. Согласно $(3) h(f)=h\left(f_{0}\right)$.

(5) Возьмем целое число $k \geqslant 2$ такое, что $k>\operatorname{deg}(f)$. Пусть $h^{*}: R \rightarrow R / x^{k} R-$ естественный эпиморфизм. Тогда $h^{*}(f)$ - центральный идемпотент кольца $h^{*}(R)$. Применим (4) к случаю $h=h^{*}$. Согласно (4) $f_{0}=\varphi\left(f_{0}\right) \in A$ и $h^{*}(f)=h^{*}\left(f_{0}\right)$. Тогда $f-f_{0} \in x^{k} R$. Так как $k>\operatorname{deg}\left(f-f_{0}\right)$, то $f=f_{0} \in A$.

(6) следует из (4) и (5).

(7) Допустим противное. Тогда $\varphi(A) a \cap \varphi(A)=0$ для некоторого ненулевого $a \in A$. Достаточно доказать, что бесконечная сумма $\sum_{n=0}^{\infty} R x^{n+1} \varphi^{n}(a)$ является прямой суммой. Для каждого $n$ все коэффициенты всех многочленов из $R x^{n+1} \varphi^{n}(a)$ содержатся в $\varphi^{n+1}(A) \varphi^{n}(a)$. Остается доказать, что сумма аддитивных групп $\varphi^{i+1}(A) \varphi^{i}(a)$ является прямой суммой. Допустим противное. Тогда $\sum_{i=k}^{n} b_{i}=0$ для некоторого $b_{i} \in \varphi^{i+1}(A) \varphi^{i}(a)$, и можно считать, что $b_{k} \neq 0$. Тогда

$$
b_{k} \in \sum_{i=k+1}^{n} \varphi^{i+1}(A) \varphi^{i}(a) \subseteq \varphi^{k+1}(A) .
$$

Поэтому $\varphi^{k+1}(A) \varphi^{k}(a) \cap \varphi^{k+1}(A) \neq 0$; получено противоречие.

(8) Так как $\varphi(A)$ - полупростое артиново кольцо, то $\varphi(A) \varphi(A)$ - прямое слагаемое модуля $\varphi(A) A$. Согласно $(2) \quad \varphi(A) \varphi(A)-$ существенньй подмодуль в $\varphi(A) A$. Поэтому $\varphi(A)=A$.

ЛЕмма 1.9. Пусть $\varphi-$ инбективный әндоморфизм кольиа $A, R \equiv A_{r}[x, \varphi], n-$ натуральное число $\geqslant 2, h: R \rightarrow R / x^{n} R$ - естественный эпиморфизм, $\mathscr{E}(A), \mathscr{E}(R) u$ $\mathscr{E}(h(R))$ - мнохества всех пирсовских слоев колеи, $A, R$ и $h(R)$ соответственно. Допустим, что $\varphi(e)=е$ для каждого центрального идемпотента е кольца $A$.

(1) Для кажсдого пирсовского слоя $A / P$ кольиа $R / P R$ u $h(R) / h(P R)$ являются пирсовскими слоями колеи, $R$ и $h(R)$ соответственно. Кроме того, правилами 
$s(A / P)=R / P R$ и $t(A / P)=h(R) / h(P R)$ задаются биекиии $s: \mathscr{E}(A) \rightarrow \mathscr{E}(R) u$ $t: \mathscr{E}(A) \rightarrow \mathscr{E}(h(R))$.

(2) Если $A / P$ - пирсовский слой кольца $A$, mо $\varphi(P) \subseteq P, \varphi$ индуиирует инбективный әндоморфизм $\bar{\varphi}$ кольиа $A / P$ и $\bar{\varphi}$ индуиирует естественные кольцевые изоморфизмы

$$
\alpha: R / P R \rightarrow(A / P)_{r}[x, \bar{\varphi}]
$$

$u$

$$
\beta: h(R) / h(P R) \rightarrow(A / P)_{r}[x, \bar{\varphi}] / x^{n}(A / P)_{r}[x, \bar{\varphi}]
$$

(3) Пусть $\mathscr{F}$ - класс колец такой, что $X \in \mathscr{F} \Longleftrightarrow$ все пирсовские слои кольиа $X$ принадлежат $\mathscr{F}$. Тогда $h(R) \in \mathscr{F} \Longleftrightarrow$ для кажддого пирсовского слоя $A / P$ кольиа $A$ кольио $(A / P)_{r}[x, \bar{\varphi}] / x^{n}(A / P)_{r}[x, \bar{\varphi}]$ принадлежит $\mathscr{F}$. Кроме того, $R \in \mathscr{F} \Longleftrightarrow$ для каждого пирсовского слоя $A / P$ кольца $A$ кольцо $(A / P)_{r}[x, \bar{\varphi}]$ принадлежит $\mathscr{F}$.

(4) $R$ - правое кольио Безу кольио $(A / P)_{r}[x, \bar{\varphi}]$ является правым кольчом Безу.

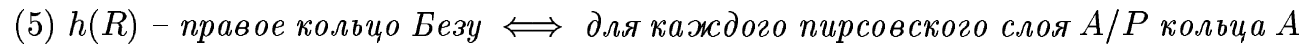
кольио $(A / P)_{r}[x, \bar{\varphi}] / x^{n}(A / P)_{r}[x, \bar{\varphi}]$ является правым кольцом Безу.

(6) Если $A$ - полупростое артиново кольцо, то $R$ - кольцо главных правых идеалов.

ДоКАЗАТЕЛЬСТво. (1) следует из леммы 1.8(6).

(2) Пусть $P=\sum_{i \in I} e_{i} A$, где все $e_{i}$ - центральные идемпотенты в $A$. Так как $\varphi\left(e_{i}\right)=e_{i}$ для всех $e_{i}$, то верно включение $\varphi(P) \subseteq P$ и $\varphi$ индуцирует эндоморфизм $\bar{\varphi}$ кольца $A / P$. Пусть $a+P \in \operatorname{Ker}(\bar{\varphi})$, где $a \in A$. Тогда $\varphi(a) \in P$ и существует конечное подмножество $J \subseteq I$ такое, что $\varphi(a) \in \sum_{j \in J} e_{j} A$. Обозначим через $u$ центральньй идемпотент кольца $A$, являюшийся произведением всех центральных идемпотентов $\left(1-e_{j}\right)(j \in J)$. Тогда $1-u \in P, \varphi(a) u=0$ и $u=\varphi(u)$ по условию. Поэтому $0=\varphi(a) \varphi(u)=\varphi(a u)$. Тогда $u a=a u \in \operatorname{Ker}(\varphi)=0$. Поэтому $a=(1-u) a \in P$ и $\bar{\varphi}-$ инъективньй эндоморфизм. Непосредственно проверяется, что $\bar{\varphi}$ индуцирует естественные кольцевые изоморфизмы $\alpha: R / P R \rightarrow(A / P)_{r}[x, \bar{\varphi}]$ и $\beta: h(R) / h(P R) \rightarrow(A / P)_{r}[x, \bar{\varphi}] / x^{n}(A / P)_{r}[x, \bar{\varphi}]$.

(3) следует из (1) и (2).

(4) и (5) следуют из (3) и леммы 1.7.

(6) Существуют ненулевые ортогональные центральные идемпотенты $e_{1}, \ldots, e_{n} \in A$ такие, что все $e_{i} A$ - простые артиновы кольца. По условию $\varphi\left(e_{i}\right)=e_{i}$ для всех $i$. Поэтому можно считать, что $A$ - простое артиново кольцо. Тогда $R$ - кольцо главных правых идеалов $[4,3.1]$.

Лемма 1.10. Пусть $A-$ риккартово справа нормальное кольцо, $\varphi-$ инбективный эндоморфизм кольца $A$ такой, что $\varphi(e)=е$ для каждого иентрального идемпотента $е \in A$ и әлемент $\varphi(d)$ обратим в кольие $A$ для каждого регулярного әлемента $d \in A$.

(1) $A_{r}[x, \varphi]-$ редуцированное кольцо.

(2) Если $A / P$ - пирсовский слой кольца $A$ u $h: A \rightarrow A / P$ - естественный эпиморфизм, то $h(A)$ - область $, \varphi(P) \subseteq P, \varphi$ индуиирует инвективный әндоморфизм $\bar{\varphi}$ области $h(A)$ и $\bar{\varphi}(\bar{a}) h(A)=h(A)$ для каждого ненулевого $\bar{a} \in h(A)$. 
ДокАЗАТЕЛьСтво. (1) Допустим противное. Положим $R \equiv A_{r}[x, \varphi]$. Существует ненулевой многочлен $f \in R$ такой, что $f^{2}=0$. Пусть $f=x^{n}(a+x g)$, где $n \geqslant 0$, $g \in R, 0 \neq a \in A$. Тогда $0=f^{2}=x^{n}(a+x g) x^{n}(a+x g)=x^{2 n}\left(\varphi^{n}(a) a+x h\right)$, где $h \in R$. Поэтому $\varphi^{n}(a) a=0$. Так как $A$ - нормальное риккартово справа кольцо, то по лемме $1.1(2) \quad a=e d$, где $e$ - ненулевой центральный идемпотент и $d$ - регулярный элемент. Кроме того, по условию $\varphi(e)=e \neq 0$. Поэтому $0=\varphi^{n}(e d) e d=\varphi^{n}(d) d e$. Кроме того, либо $n=0$ и $\varphi^{n}(d)=d$, либо $n>0$ и $\varphi^{n}(d)$ - обратимьй элемент по условию. В обоих случаях $\varphi^{n}(d) d$ - регулярный элемент. Так как $\varphi^{n}(d) d e=0$, то верно равенство $e=0$. Получено противоречие.

(2) По лемме 1.1(5) $h(A)$ - область. По лемме $1.9(2) \quad \varphi(P) \subseteq P$ и $\varphi$ индуцирует инъективньй эндоморфизм $\bar{\varphi}$ области $h(A)$. Пусть $\bar{a}=h(a)$, где $a \in A \backslash P$. Так как $A-$ риккартово справа нормальное кольцо, то по лемме 1.1(2) $a=e d$, где $e$ - центральньй идемпотент кольца $A, d$ - регулярньй элемент. Поскольку $\bar{a}=h(e) h(d) \neq 0$, то $h(e)-$ ненулевой идемпотент области $h(A)$. Поэтому $h(e)=h(1)$ и $h(e A)=h(A)$. По условию $\varphi(e)=е$ и $\varphi(d) A=A$. Поэтому $\bar{\varphi}(\bar{a}) h(A)=h(e) h(\varphi(d) A)=h(e A)=h(A)$.

ДоказАтЕльство теоремы 1 . Так как в кольце $A$ все правые аннуляторы являются идеалами, то $A-$ нормальное кольцо. Импликация $(3) \Longrightarrow(1)$ очевидна. Импликация $(1) \Longrightarrow(2)$ следует из того, что все факторкольца правых колец Безу являются правыми кольцами Безу.

$(2) \Longrightarrow(4)$. По лемме $1.3 A$-правое кольцо Безу и $a \in a \varphi(a) A$ для любого $a \in A$. По лемме $1.2 A$ - риккартово справа правое кольцо Безу, $\varphi(e)=e$ для каждого центрального идемпотента $e \in A$ и элемент $\varphi(a)$ обратим в $A$ для каждого регулярного элемента $a \in A$.

$(4) \Longrightarrow(3)$. По лемме $1.10(1) A_{r}[x, \varphi]$ - редуцированное кольцо. Пусть $A / P$ - пирсовский слой кольца $A, h: A \rightarrow A / P$ - естественньй эпиморфизм. По лемме $1.10(2)$ $h(A)$ - область, $\varphi(P) \subseteq P, \varphi$ индуцирует инъективньй эндоморфизм $\bar{\varphi}$ области $h(A)$ и $\bar{\varphi}(\bar{a}) h(A)=h(A)$ для каждого ненулевого $\bar{a} \in h(A)$. Так как $A-$ правое кольцо Безу, то $h(A)$ - правое кольцо Безу. По лемме $1.5 h(A)_{r}[x, \bar{\varphi}]$ - правое кольцо Безу. По лемме $1.9(4) A_{r}[x, \bar{\varphi}]$ - правое кольцо Безу.

\section{2. Доказательство теорем 2, 3 и 4.}

Лемма 2.1. Пусть $A-$ кольио.

(1) Если $A$ - дистрибутивное справа кольцо, то $A$ - нормальное кольцо и все его нильпотентные әлементы лежсат в $J(A)$.

(2) Если $A$ - квази-инвариантное справа правое кольцо Безу, то А дистрибутивно справа.

(3) Если $A / J(A)$ - строго регулярное кольцо, то $A-$ правое кольцо Безу $\Longleftrightarrow$ А дистрибутивно справа.

(4) Если существует нильидеал $N$ кольца $A$ такой, что $A / N$ - строго регулярное кольцо, то $A-$ правое кольио Безу $А$ дистрибутивно справа.

(5) Для любих $a, b \in A$ существуют $c, d, f, g \in A$ такие, ито $1=c+d, a c=b f$,

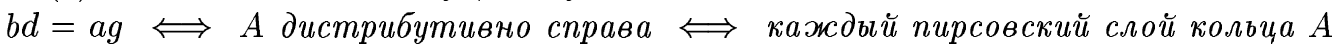
дистрибутивен.

ДокАЗАТЕЛЬСТво. (1) доказано в $[1,5.14(1), 8.35(4)] ;(2)$ и (3) доказаны в $[1,3.26$, $3.33]$. 
(4) Так как $N$ - нильидеал, то $N \subseteq J(A)$. Поэтому кольцо $A / J(A)$ изоморфно факторкольцу строго регулярного кольца $A / N$. Тогда $A / J(A)$ - строго регулярное кольцо и (4) следует из (3).

(5) Первая эквивалентность доказана в $[1,1.17]$, вторая эквивалентность доказана в $[3,6.94]$.

Лемма 2.2. Пусть $A-$ строго регулярное кольио.

(1) А - инвариантное дистрибутивное редуцированное риккартово кольио Безу, в котором каждый әлемент является произведением иентрального идемпотента на обратимый әлемент.

(2) Если $d$ - регулярный справа әлемент кольиа $A$ и $\varphi$ - инвективный әндоморфизм кольиа $A$, то $d u \varphi(d)$ - обратимые әлементы.

ДокАЗАТЕЛЬСТво. (1) хорошо известно (см., например, $[1,3.30]) ;(2)$ следует из (1).

Лемма 2.3. Пусть $A$ - строго регулярное кольио, $\varphi-$ инвективный әндоморфизм кольца $A, n$ - натуральное число, $R \equiv A_{r}[x, \varphi], h: R \rightarrow R / x^{n} R$ - естественный эпиморфизм.

(1) $h(R)$ - правое кольио Безу $h(R)$ дистрибутивно справа.

(2) Eсли $\varphi(e)=е$ для каждого иентрального идемпотента е кольца $A$, то для каждого главного правого идеала $T$ кольца $R$ существуют ортогональные идемпотенты $e_{0}, \ldots, e_{n-1} \in A$ такие, что все $e_{i}$ лежсат в иентре кольца $R u$

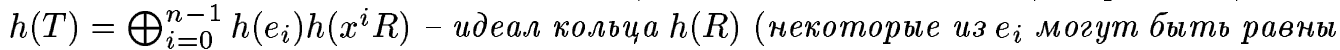
нулю).

ДокАЗАТЕЛьство. (1) Идеал $h(x R)$ кольца $h(R)$ нильпотентен. Так как $h(R) / h(x R)$ $\cong A$, то $h(R) / h(x R)$ - строго регулярное кольцо. Поэтому (1) следует из леммы $2.1(4)$.

(2) Положим $T_{0} \equiv T$. Пусть $T_{0}=f^{(0)} R \neq 0$ и $f^{(0)}=a_{0}+x g^{(0)}$, где $a_{0} \in A$ и $g^{(0)} \in R$. По лемме $2.2(3) \quad a_{0}=e_{0} d_{0}$, где $e_{0}$ - центральньй идемпотент и $d_{0}-$ обратимый элемент. Так как $\varphi\left(e_{0}\right)=e_{0}$, то $e_{0}$ - центральный идемпотент кольца $R$ и $T_{0}=e_{0}\left(d_{0}+x g^{(0)}\right) R \oplus x\left(1-e_{0}\right) g^{(0)} R$. Положим $f^{(1)} \equiv\left(1-e_{0}\right) g^{(0)} \in R, T_{1} \equiv x f^{(1)} R$. Пусть $f^{(1)}=a_{1}+x g^{(1)}$, где $a_{1} \in A$ и $g^{(1)} \in R$. По лемме 2.2(3) $a_{1}=e_{1} d_{1}$, где $e_{1}-$ центральньй идемпотент и $d_{1}$ - обратимьй элемент. Так как $\varphi\left(e_{1}\right)=e_{1}$, то $e_{1}-$ центральньй идемпотент кольца $R$ и $T_{1}=e_{1} x\left(d_{1}+x g^{(1)}\right) R \oplus x^{2}\left(1-e_{1}\right) g^{(1)} R$. Положим $f^{(2)} \equiv\left(1-e_{1}\right) g^{(1)} \in R, T_{2} \equiv x f^{(2)} R$. Повторим эти действия несколько раз. Так как многочлен $f^{(0)}$ имеет конечную степень, то в конце концов мы получим прямое разложение

$$
T=\bigoplus_{i=0}^{n-1} e_{i} x^{i}\left(d_{i}+x g^{(i)}\right) R
$$

где $g^{(i)} \in R, d_{i}$ - обратимые элементы кольца $A, e_{i}$ - ортогональные идемпотенты кольца $A$, лежащие в центре кольца $R(i=0, \ldots, n-1)$. Так как $h\left(x g^{(i)}\right)$ - нильпотентные элементы кольца $h(R)$ и $h\left(d_{i}\right)$ - обратимые элементы кольца $h(A)$, то $h\left(d_{i}+x g^{(i)}\right)-$ обратимые элементы кольца $h(R)$ для всех $i$. Поэтому $h\left(x^{i}\left(d_{i}+x g^{(i)}\right) R\right)=h\left(x^{i} R\right)$ для всех $i$. Кроме того, $h\left(e_{i}\right)$ - ортогональные идемпотенты кольца $h(A)$, лежащие в центре кольца $h(R)(i=0, \ldots, n-1)$. Тогда

$$
h(T)=\bigoplus_{i=0}^{n-1} h\left(e_{i}\right) h\left(x^{i}\left(d_{i}+x g^{(i)}\right) R\right)=\bigoplus_{i=0}^{n-1} h\left(e_{i}\right) h\left(x^{i} R\right)
$$


- идеал кольца $h(R)$.

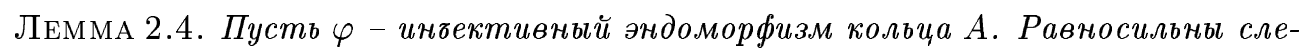
дующие условия:

(1) $A_{r}[x, \varphi]-$ нормальное кольчо;

(2) $A_{r}[x, \varphi] / x^{2} A_{r}[x, \varphi]$ - нормальное кольцо;

(3) $A_{r}[x, \varphi] / x^{n} A_{r}[x, \varphi]$ - нормальное кольцо для каждого натурального числа $n$;

(4) $A$ - нормальное кольцо и $\varphi(e)=е$ для каждого иентрального идемпотента $e \in A$.

ДокАЗАТЕЛЬСтво. Положим $R \equiv A_{r}[x, \varphi]$. Импликация $(3) \Longrightarrow(2)$ очевидна.

$(2) \Longrightarrow(4)$. Пусть $h: R \rightarrow R / x^{2} R$-естественньй эпиморфизм. Так как $A \cap \operatorname{Ker}(h)=0$, то кольцо $A$ изоморфно подкольцу нормального кольца $h(R)$. Поэтому $A$ - нормальное кольцо. Пусть $e$-центральньй идемпотент кольца $A$. Тогда $h(e)$-идемпотент нормального кольца $h(R)$. Поэтому $h(e x)=h(e) h(x)=h(x) h(e)=h(x e)$. С другой стороны, $h(e x)=h(x \varphi(e))$. Тогда $h(x \varphi(e)-x e)=0$, откуда $x(\varphi(e)-e) \in x^{2} R$. Поэтому $\varphi(e)-e=0$.

$(4) \Longrightarrow(3)$. Пусть $h: R \rightarrow R / x^{n} R$ - естественный эпиморфизм и $f-$ многочлен из $R$ такой, что $h(f)$ - ненулевой идемпотент кольца $h(R)$. Пусть $e-$ свободньй член многочлена $f$. По лемме $1.8(1) \quad e$ - ненулевой идемпотент нормального кольца $A$. По условию $\varphi(e)=e$. Тогда $e x=x e$. Поэтому $e-$ центральньй идемпотент кольца $R$. Тогда $h(e)$ - центральньй идемпотент кольца $h(R)$. По лемме $1.8(3) h(f)=h(e)$.

$(1) \Longleftrightarrow(4)$. Доказательство импликации $(1) \Longrightarrow(4)$ аналогично доказательству импликации $(2) \Longrightarrow(4)$. Доказательство импликации $(4) \Longrightarrow(1)$ аналогично доказательству импликации $(4) \Longrightarrow(3)$.

ДОКАЗАТЕЛЬСТВО ТЕОРЕМЫ 2. ИМпликации (5) $\Longrightarrow(4)$ и (5) $\Longrightarrow(1)$ очевидны. Эквивалентность условий (3) и (4) следует из леммы $2.3(1)$. Импликация $(1) \Longrightarrow(2)$ следует из того, что каждое инвариантное справа кольцо является нормальным кольцом. Импликация (2) $\Longrightarrow(6)$ следует из леммы 2.4. Импликация $(4) \Longrightarrow(2)$ следует из того, что каждое дистрибутивное справа кольцо нормально по лемме 2.1(1).

$(6) \Longrightarrow(5)$. По лемме $2.2 A$-риккартово кольцо Безу, причем элемент $\varphi(d)$ обратим в $A$ для каждого регулярного элемента $d \in A$. Пусть $R \equiv A_{r}[x, \varphi], h: R \rightarrow R / x^{k} R-$ естественный эпиморфизм. По теореме $1 R$-редуцированное правое кольцо Безу. Тогда $h(R)$ - правое кольцо Безу. По лемме $2.3(2)$ каждый главный правый идеал кольца $h(R)$ является идеалом в $h(R)$. Поэтому $h(R)$ - инвариантное справа кольцо. По лемме 2.1(2) инвариантное справа правое кольцо Безу $h(R)$ дистрибутивно справа.

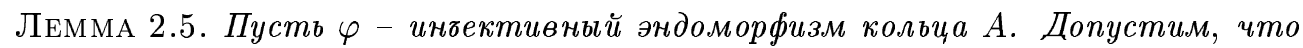
$A_{r}[x, \varphi] / x^{2} A_{r}[x, \varphi]-$ дистрибутивное справа кольио.

(1) $A$ дистрибутивно справа и для любого $а \in A$ существует әлемент $b \in A$ такой, что $a=a \varphi(a) b$.

(2) $A$ - нормальное кольцо, $\varphi(e)=е$ для любого идемпотента $е \in A$ и для каждого регулярного справа әлемента $d \in A$ әлемент $\varphi(d)$ обратим в кольце $A$.

(3) A - риккартово редуцированное кольио. 
ДокАЗАТЕЛЬСТво. Пусть $R \equiv A_{r}[x, \varphi]$ и $h: R \rightarrow R / x^{2} R$ - естественньй эпиморфизм.

(1) Так как $A \cong h(R) / h(x R)$, то $A$ - дистрибутивное справа кольцо. Пусть $a \in A$. По лемме $2.1(5)$ для элементов $h(a)$ и $h(x)$ дистрибутивного справа кольца $h(R)$ существуют многочлены $f, c, d \in R$ такие, что $h(a) h(f)=h(x) h(c)$ и $h(x) h(1-f)=h(a) h(d)$. Поэтому существуют многочлены $v, w \in R$ такие, что $a f=x c+x^{2} v$ и $x(1-f)=a d+x^{2} w$. Приравнивая свободные члены в первом равенстве и коэффициенты при $x$ во втором равенстве, получим равенства $a f_{0}=0$ и $1-f_{0}=\varphi(a) d_{1}$, где $f_{0}-$ свободный член многочлена $f$ и $d_{1} \equiv b$ - коэффициент при $x$ многочлена $d$. Поэтому $a=a\left(1-f_{0}\right)=a \varphi(a) b$.

(2) По лемме 2.1(1) дистрибутивное справа кольцо $A$ является нормальным кольцом. Поэтому оставшиеся утверждения следуют из (1), леммы 1.1(4) и леммы 1.1(6).

(3) Пусть $a$ - произвольньй нильпотентньй элемент кольца $A$. Согласно $(1)$ существует элемент $b \in A$ такой, что $a(1-\varphi(a) b)=0$. Так как $\varphi(a)$ - нильпотентньй элемент, то $\varphi(a) \in J(A)$ по лемме 2.1(1). Поэтому $1-\varphi(a) b$ - обратимьй элемент. Тогда $0=a(1-\varphi(a) b)(1-\varphi(a) b)^{-1}=a$ и $A-$ редуцированное кольцо. По лемме $1.1(1)$ все правые аннуляторы элементов из $A$ являются идеалами в $A$. По лемме $1.2(2) A$ - риккартово кольцо.

ЛЕмма 2.6. Пусть $\varphi$ - инбективный әндоморфизм кольиа $A, R \equiv A_{r}[x, \varphi], n-$ натуральное число $\geqslant 2$. Равносильны следующие условия:

(1) $R / x^{n} R$ - дистрибутивное справа кольио;

(2) для кажсдого пирсовского слоя $A / P$ кольиа $A$ эндоморфизм $\varphi$ индуциру-

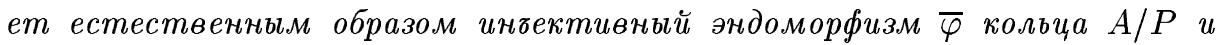
$(A / P)_{r}[x, \bar{\varphi}] / x^{n}(A / P)_{r}[x, \bar{\varphi}]-$ дистрибутивное справа кольио.

ДокАЗАтЕльство. Пусть $\mathscr{F}$ - класс всех дистрибутивных справа колец. По лемме 2.1(5) $X \in \mathscr{F} \Longleftrightarrow$ все пирсовские слои кольца $X$ принадлежат $\mathscr{F}$. Пусть $e-$ произвольный центральньй идемпотент кольца $A$. Если $\varphi(e)=e$, то эквивалентность $(1) \Longleftrightarrow(2)$ следует из леммы $1.9(3)$. Поэтому достаточно доказать, что $\varphi(e)=e$, если выполняется (1) или (2).

Допустим, что вьполнено (1). Тогда кольцо $R / x^{2} R$ дистрибутивно справа, поскольку $R / x^{2} R$ - гомоморфный образ дистрибутивного справа кольца $R / x^{n} R$. По лемме $2.5(2)$ $\varphi(e)=e$.

Допустим, что выполнено (2). Пусть $A / P$ - любой пирсовский слой кольца $A$ и $\pi: A \rightarrow$ $A / P$ - естественный эпиморфизм. Так как каждое кольцо является подпрямым произведением своих пирсовских слоев $[3,6.89(10)]$, то достаточно доказать, что $\varphi(e)-e \in P$. Так как $(A / P)_{r}[x, \bar{\varphi}] / x^{2}(A / P)_{r}[x, \bar{\varphi}]$ - гомоморфньй образ дистрибутивного справа кольца $(A / P)_{r}[x, \bar{\varphi}] / x^{n}(A / P)_{r}[x, \bar{\varphi}]$, то $(A / P)_{r}[x, \bar{\varphi}] / x^{2}(A / P)_{r}[x, \bar{\varphi}]$ - дистрибутивное справа кольцо. По лемме $2.5(2) \bar{\varphi}(\pi(e))=\pi(e)$. Поэтому $\varphi(e)-e \in P$.

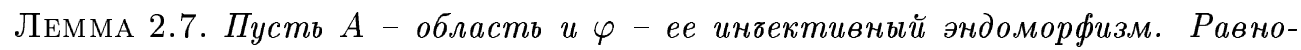
сильны следующие условия:

(1) $A_{r}[x, \varphi] / x^{2} A_{r}[x, \varphi]-$ дистрибутивное справа кольио;

(2) $A_{r}[x, \varphi] / x^{n} A_{r}[x, \varphi]$ - дистрибутивное справа кольцо для каждого натурального числа $n$;

(3) А дистрибутивно справа и $\varphi(a) A=A$ для кажсого ненулевого а $\in A$. 
ДокАЗАТЕЛЬСтво. Импликация $(2) \Longrightarrow(1)$ очевидна. Импликация $(1) \Longrightarrow$ следует из леммы 2.5.

$(3) \Longrightarrow(2)$. Положим $R \equiv A_{r}[x, \varphi]$. Пусть $h: R \rightarrow R / x^{n} R$ - естественньй эпиморфизм, $F, G$ и $H$ - произвольные главные правые идеалы кольца $R$. Достаточно доказать следующее равенство:

$$
h(F) \cap(h(G)+h(H))=h(F) \cap h(G)+h(F) \cap h(H) .
$$

Если любые два из трех правых идеалов $h(F), h(G), h(H)$ не сравнимы по включению, то равенство (*) следует из леммы 1.4(5). Если либо $h(F) \subseteq h(G)$, либо $h(F) \subseteq h(H)$, то равенство $(*)$ проверяется непосредственно. Если либо $h(F) \supseteq h(G)$, либо $h(F) \supseteq h(H)$, то равенство $(*)$ следует из модулярного закона.

ДоКАЗАТЕЛЬСТВо ТЕОРЕМЫ 3. Так как все гомоморфные образы дистрибутивных справа колец являются дистрибутивными справа кольцами, то импликация $(2) \Longrightarrow(1)$ следует из того, что $A_{r}[x, \varphi] / x^{2} A_{r}[x, \varphi]$ - гомоморфньй образ кольца $A_{r}[x, \varphi] / x^{n} A_{r}[x$, $\varphi]$, если $n \geqslant 2$. Импликация $(1) \Longrightarrow(3)$ следует из леммы 2.5 .

$(3) \Longrightarrow(2)$. Дистрибутивное справа кольцо $A$ является нормальным кольцом по лемме 2.1(1). Пусть $A / P$ - произвольньй пирсовский слой кольца $A$ и $h: A \rightarrow A / P$ - естественньй эпиморфизм. По лемме $1.10(2) h(A)$ - область, $\varphi(P) \subseteq P, \varphi$ индуцирует инъективньй эндоморфизм $\bar{\varphi}$ области $h(A)$ и $\bar{\varphi}(\bar{a}) h(A)=h(A)$ для каждого ненулевого $\bar{a} \in h(A)$. По лемме $2.7 h(A)_{r}[x, \bar{\varphi}] / x^{n} h(A)_{r}[x, \bar{\varphi}]$ - дистрибутивное справа кольцо. По лемме $2.6 A_{r}[x, \varphi] / x^{n} A_{r}[x, \varphi]$ - дистрибутивное справа кольцо.

ЛЕмма 2.8. (1) Каждое ортогонально конечное кольцо является прямо-конечнblм.

(2) Если каждое примитивное справа факторкольцо кольиа А либо ортогонально конечно, либо прямо-конечно, то $A$ - прямо-конечное кольчо.

(3) Если $A$ - РІ-кольцо, то $A$ - прямо-конечное кольцо, у которого все примитивные справа факторкольиа являются артиновыми кольиами.

(4) Если $A$ - бирегулярное кольцо, то все пирсовские слои кольца $A$ являются простымми кольцами.

ДокАЗАТЕЛЬСТво. (1) доказано в $[5,19.40]$.

(2) Пусть $a, b \in A$ и $a b=1$. Достаточно доказать, что $b a-$ обратимьй элемент. Положим $x \equiv 1-b a$. Если $x \in J(A)$, то $b a=1-x$-обратимьй элемент. Допустим, что $x \notin J(A)$. Тогда существует примитивное справа факторкольцо $A / P$ такое, что $h(x) \neq 0$, где $h: A \rightarrow A / P$ - естественный эпиморфизм. Согласно (1) $h(A)-$ прямо-конечное кольцо. Так как $h(a) h(b)=h(1)$, то $h(b) h(a)=h(1)$. С другой стороны, $h(b) h(a) \neq h(1)$, поскольку $h(1)-h(b) h(a)=h(x) \neq 0$. Получено противоречие.

(3) следует из (2) и того, что каждое примитивное справа PI-кольцо является артиновым кольцом.

(4) Пусть $A / P$ - произвольньй пирсовский слой кольца $A, h: A \rightarrow A / P$ - естественный эпиморфизм, $a$ - элемент кольца $A$ такой, что $h(a)$ - ненулевой элемент кольца $h(A)$. Так как $A$ - бирегулярное кольцо, то существует центральный идемпотент $e$ кольца $A$ такой, что $A a A=e A$. Тогда $h(e A)=h(A a A) \neq 0$ и $e \notin P$. Так как $A / P-$ пирсовский слой кольца $A$ и $e$ - центральньй идемпотент кольца $A$, не лежащий в идеале $P$, то $h(1)=h(e) \in h(A a A)$. Поэтому $A / P-$ простое кольцо. 
ЛЕмма 2.9. Пусть $A$ - прямо-конечное кольцо, $\varphi-$ инбективный эндоморфизм кольиа $A$. Допустим, что $A_{r}[x, \varphi] / x^{2} A_{r}[x, \varphi]-$ правое кольио Безу.

(1) $\varphi(e)=е$ для каждого чентрального идемпотента $е \in A$.

(2) Для каждого регулярного справа әлемента $d \in A$ әлемент $\varphi(d)$ обратим в $A$.

(3) Верно равенство $\varphi(A) \cap J(A)=0$. Следовательно, если $\varphi-$ автоморфизм, то $A$ полупримитивно.

(4) Если ч отображсает первичный радикал $N$ кольца $A$ внутрь $J(A)$, то $A$ полупервично. Следовательно, если $\varphi(N) \subseteq N$, то $A$ полупервично.

(5) Если $\varphi-$ автоморфизм и $B$ - идеал кольиа $A$ такой, что $\varphi(B)=B$, то $B^{2}=B$ и $A / B$ - полупримитивное кольио.

ДокАЗАтЕЛьство. (1) Пусть $e$ - центральный идемпотент кольца $A$. Из леммы 1.3(1) и того, что $e$ - центральный идемпотент, следует существование элементов $f_{0}, g_{0}, u_{0}, v_{0}, b, c \in A$ таких, что вьполнены следующие равенства:

(i) $e=\left(e f_{0}\right)\left(e u_{0}\right)$;

(ii) $\left(e f_{0}\right)\left(e v_{0}\right)=0$;

(iii) $1=\varphi(e) b+g_{0} v_{0}$.

Так как $A$ - прямо-конечное кольцо, то $e A$ - прямо-конечное кольцо. Поэтому из (i) следует, что элементы $e f_{0}$ и $e u_{0}$ обратимы в кольце $e A$. Тогда из (ii) следует, что $0=e v_{0}=$ $v_{0} e$. Поэтому из (iii) следует, что $e=e \varphi(e) b$. Следовательно, $e=e \varphi(e)$ для произвольного центрального идемпотента $e \in A$. Так как $1-e$ также центральный идемпотент, то аналогично получаем, что $1-e=(1-e) \varphi(1-e)=(1-e)(1-\varphi(e))$. Тогда $\varphi(e)=e \varphi(e)+(1-e) \varphi(e)=e+(1-e)(1-\varphi(e)) \varphi(e)=e$.

$(2)$ Из леммы $1.3(1)$ и равенства $r(d)=0$ следует существование $f_{0}, g_{0}, u_{0}, v_{0}, b \in A$ таких, что $1=f_{0} u_{0}, f_{0} v_{0}=0,1=\varphi(d) b+g_{0} v_{0}$. Так как $A$ - прямо-конечное кольцо, то из равенства $1=f_{0} u_{0}$ следует, что $f_{0}$ - обратимьй элемент. Так как $f_{0} v_{0}=0$, то $v_{0}=0$. Поэтому из равенства $1=\varphi(d) b+g_{0} v_{0}$ следует, что $1=\varphi(d) b$. Так как $A-$ прямо-конечное кольцо, то $\varphi(d)$ - обратимый элемент.

(3) Пусть $a \in A$ и $\varphi(a) \in J(A)$. По лемме $1.3(1)$ существуют $f_{0}, g_{0}, u_{0}, v_{0}, b \in A$ такие, что верны следующие равенства:

(i) $a=a f_{0} u_{0}$;

(ii) $a f_{0} v_{0}=0$;

(iii) $1=\varphi(a) b+g_{0} v_{0}$.

Так как $\varphi(a) \in J(A)$, то элемент $1-\varphi(a) b$ обратим. В силу (iіi) элемент $g_{0} v_{0}$ обратим. Тогда элемент $v_{0}$ обратим слева. Так как $A$-прямо-конечное кольцо, то $v_{0}$ - обратимый элемент. Поэтому из (ii) следует, что $a f_{0}=0$. По (i) $a=a f_{0} u_{0}=0$.

(4) Согласно (3) $\varphi(A) \cap J(A)=0$. Так как $\varphi(N) \subseteq J(A), \operatorname{то} \varphi(N) \subseteq \varphi(A) \cap J(A)=0$. Поэтому $N=0$.

(5) Пусть $h: A \rightarrow A / B^{2}$ - естественньй эпиморфизм. Так как $\varphi(B)=B$, то $\varphi\left(B^{2}\right)=$ $B^{2}$ и автоморфизм $\varphi$ индуцирует автоморфизм $\bar{\varphi}$ факторкольца $h(A)$. Так как кольцо $h(A)_{r}[x, \bar{\varphi}] / x^{2} h(A)_{r}[x, \bar{\varphi}]$ является гомоморфньп образом правого кольца Безу $A_{r}[x, \varphi] /$ $x^{2} A_{r}[x, \varphi]$, то $h(A)_{r}[x, \bar{\varphi}] / x^{2} h(A)_{r}[x, \bar{\varphi}]$ - правое кольцо Безу. Применяя $(3)$ к автоморфизму $\bar{\varphi}$ кольца $h(A)$, получим, что $h(A)$ - полупримитивное кольцо. В частности, $h(A)$ - полупервичное кольцо с нильпотентным идеалом $h(B)$. Тогда $h(B)=0$, откуда $B=B^{2}$ и $h(A)=A / B$. 
Лемма 2.10. Пусть $A$ - такое кольцо, что $A[x] / x^{2} A[x]-$ правое кольио Безу.

(1) Если $A$ прямо-конечно, то $B^{2}=B$ для каждого его идеала $B$.

(2) Если каждое примитивное справа факторкольцо кольиа $A$ ортогонально конечно, то $B^{2}=B$ для каждого идеала $B$ кольца $A$.

(3) Если $A$ - PI-кольио, то $A$-регулярное кольио.

ДокАЗАТЕЛЬСТво. (1) следует из леммы $2.9(5)$ при $\varphi \equiv 1_{A} ;(2)$ следует из (1) и леммы 2.8(2).

(3) Из (2) и леммы $2.8(3)$ следует, что $B^{2}=B$ для каждого идеала $B$ кольца $A$. Так как $A$ - РІ-кольцо и $B^{2}=B$ для каждого его идеала $B$, то $A$ регулярно [6, теорема 1$]$.

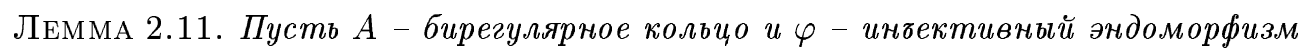
кольиа $A$ такой, что $\varphi(e)=е$ для каждого иентрального идемпотента $е \in A$.

(1) Если все пирсовские слои кольца $A$ являются артиновыми кольцами, то $A_{r}[x, \varphi]-$ правое кольцо Безу.

(2) Если $A$ - PІ-кольио, то $A_{r}[x, \varphi]-$ правое кольцо Безу.

ДокАЗАТЕЛЬСТво. Пусть $A / P$ - произвольньй пирсовский слой кольца $A$. По лемме 2.8(4) $A / P$ - простое кольцо.

(1) По условию простое кольцо $A / P$ является артиновым. По лемме $1.9(6)(A / P)_{r}[x$, $\bar{\varphi}]$ - кольцо главных правых идеалов. По лемме $1.9(4) A_{r}[x, \varphi]$ - правое кольцо Безу.

(2) По лемме 2.8(3) простое РІ-кольцо $A / P$ является артиновым кольцом. Согласно (1) $A_{r}[x, \varphi]-$ правое кольцо Безу.

ДоКАЗАТЕЛЬСТво ТЕОРЕМЫ 4. Импликация (1) $\Longrightarrow(2)$ следует из того, что все факторкольца правых колец Безу являются правыми кольцами Безу. Импликация $(2) \Longrightarrow(3)$ следует из леммы $2.10(3)$ и того, что каждое кольцо, являющееся конечно-порожденньм модулем над своим центром, является РІ-кольцом.

$(3) \Longrightarrow(1)$. Так как $A$ - регулярное кольцо, являющееся конечно-порожденным модулем над своим центром, то $A$ - бирегулярное кольцо [7, теорема 6.3]. По лемме 2.11(2) $A[x]$ - правое кольцо Безу.

ПРЕДЛОЖЕНИЕ 2.12. Пусть $\varphi-$ автоморфизм кольца А. Равносильны следующие условия:

(1) $A$ - правое или левое кольцо Голди и $A_{r}[x, \varphi]$ - правое кольцо Безу;

(2) $A$ - правое или левое кольио Голди и $A_{r}[x, \varphi] / x^{2} A_{r}[x, \varphi]-$ правое кольцо Безу;

(3) $A_{r}[x, \varphi]-$ кольцо главных правых идеалов;

(4) $A_{r}[x, \varphi] / x^{2} A_{r}[x, \varphi]-$ кольцо главных правых идеалов;

(5) $A$ - артиново полупростое кольио и $\varphi(e)=е$ для каждого иентрального идемпотента е $\in$ A.

ДокАЗАТЕЛЬСТво. Импликация (5) $\Longrightarrow(3)$ следует из леммы $1.9(6)$. Импликации $(3) \Longrightarrow(4),(4) \Longrightarrow(2),(3) \Longrightarrow(1)$ и $(1) \Longrightarrow(2)$ проверяются непосредственно.

$(2) \Longrightarrow(5)$. По лемме $2.8(1) A$ - прямо-конечное кольцо. По леммам $2.9(3)$ и $2.9(1)$ $A$ - полупримитивное кольцо и $\varphi(e)=e$ для каждого центрального идемпотента $e \in A$. Так как $A$ - полупервичное правое или левое кольцо Голди, то $A$ - правьй или левьй порядок в полупростом артиновом кольце $Q$. По лемме $2.9(2)$ для каждого регулярного 
элемента $d \in A$ элемент $\varphi(d)$ обратим в $A$. Кроме того, $\varphi$ - автоморфизм. Поэтому каждьй регулярньй элемент кольца $A$ обратим в $A$. Тогда $A=Q$.

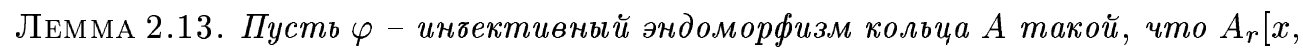
$\varphi] / x^{2} A_{r}[x, \varphi]$ - левое кольио Безу.

(1) Для каждого $а \in A$ существуют $f_{0}, g_{0}, u_{0}, v_{0}, b, c \in A$ такие, что верны следующие равенства:

(i) $a=u_{0} f_{0} a$;

(ii) $v_{0} f_{0} a=0$;

(iii) $\varphi\left(u_{0}\right) \varphi\left(g_{0}\right)=b a$;

(iv) $\varphi\left(v_{0}\right) \varphi\left(g_{0}\right)=1-c a$.

(2) Если кольио А прямо-конечно, то А полупримитивно, кажсдый его регулярный слева әлемент обратим и $\varphi(e)=е$ для кажсдого иентрального идемпотента $e \in A$.

(3) Если $A$ - правое или левое кольио Голди, то $A$ - полупростое артиново кольио и $\varphi(e)=е$ для каждого иентрального идемпотента $е \in A$.

ДокаЗАтеЛЬСтво. (1) Положим $R \equiv A_{r}[x, \varphi]$. Пусть $h: R \rightarrow R / x^{2} R$ - естественньй эпиморфизм. Так как $h(R)$ - левое кольцо Безу, то $h(R a+R x)$ - главный левый идеал кольца кольца $h(R)$. Поэтому существуют многочлены $f, g, u, v \in R$ такие, что $u(f a+g x)-a \in x^{2} R$ и $v(f a+g x)-x \in x^{2} R$. Пусть $f_{0}, g_{0}, u_{0}, v_{0}-$ свободные члены многочленов $f, g, u, v$ соответственно. Пусть $f_{1}, u_{1}, v_{1}$ - канонические коэффициенты при $x$ многочленов $f, u, v$ соответственно. Свободные члены и коэффициенты при $x$ многочленов $u(f a+g x)-a$ и $v(f a+g x)-x$ равны нулю. Поэтому получаем следующие равенства: $u_{0} f_{0} a=a, v_{0} f_{0} a=0,\left(u_{1} f_{0}+\varphi\left(u_{0}\right) f_{1}\right) a+\varphi\left(u_{0}\right) \varphi\left(g_{0}\right)=0$, $\left(v_{1} f_{0}+\varphi\left(v_{0}\right) f_{1}\right) a+\varphi\left(v_{0}\right) \varphi\left(g_{0}\right)=1$. Теперь можно положить $b \equiv-u_{1} f_{0}-\varphi\left(u_{0}\right) f_{1}$, $c \equiv v_{1} f_{0}+\varphi\left(v_{0}\right) f_{1}$.

(2) Пусть $a \in A$. Согласно (1) существуют $f_{0}, g_{0}, u_{0}, v_{0}, b, c \in A$ такие, что верны следующие равенства:

(i) $a=u_{0} f_{0} a$;

(ii) $v_{0} f_{0} a=0$;

(iii) $\varphi\left(u_{0}\right) \varphi\left(g_{0}\right)=b a$;

(iv) $\varphi\left(v_{0}\right) \varphi\left(g_{0}\right)=1-c a$.

Допустим, что $a \in J(A)$. Тогда элемент $1-c a$ обратим. В силу (iv) элемент $\varphi\left(v_{0}\right)$ обратим справа. Так как $A$ прямо-конечно, то элемент $\varphi\left(v_{0}\right)$ обратим. Кроме того, из (ii) следует равенство $\varphi\left(v_{0}\right) \varphi\left(f_{0} a\right)=0$. Поэтому $\varphi\left(f_{0} a\right)=0$, откуда $f_{0} a=0$. В силу (i) $a=u_{0}\left(f_{0} a\right)=0$. Поэтому $A$ полупримитивно.

Допустим, что $a$ - регулярный слева элемент. В силу (i) $1-u_{0} f_{0} \in \ell(a)=0$. Поэтому элемент $u_{0}$ обратим справа. Так как $A$ прямо-конечно, то $u_{0}$ обратим. Поэтому $\varphi\left(u_{0}\right)$ обратим. В силу (iii) $\varphi\left(g_{0}\right)=\left(\varphi\left(u_{0}\right)\right)^{-1} b a$. Из этого равенства и из (iv) следует равенство $1=c a+\varphi\left(v_{0}\right)\left(\varphi\left(u_{0}\right)\right)^{-1} b a$. Поэтому $a-$ обратимьй слева элемент прямо-конечного кольца $A$. Тогда $a$ обратим.

Допустим, что $a$ - центральньй идемпотент в $A$. Так как $A$ прямо-конечно, то кольцо $A a$ прямо-конечно. Согласно (i) $\left(u_{0} a\right)\left(f_{0} a\right)=a$ - единица прямо-конечного кольца $A a$. Поэтому $f_{0} a$ - обратимьй элемент кольца $A a$. Кроме того, $\left(v_{0} a\right)\left(f_{0} a\right)=0$ в силу (ii). 
Тогда $0=v_{0} a=a v_{0}$. Кроме того, $1-c a=\varphi\left(v_{0}\right) \varphi\left(g_{0}\right)$ в силу (iv). Поэтому

$$
\begin{aligned}
(1-a) \varphi(a) & =\varphi(a)(1-a)(1-c a)=(1-a) \varphi(a)(1-c a) \\
& =(1-a) \varphi(a) \varphi\left(v_{0}\right) \varphi\left(g_{0}\right)=(1-a) \varphi\left(a v_{0}\right) \varphi\left(g_{0}\right)=0
\end{aligned}
$$

Следовательно, $\varphi(a)=a \varphi(a) \in a A$. Так как $1-a-$ центральньй идемпотент и $\varphi(a) \in A a$ для произвольного центрального идемпотента $a \in A$, то $\varphi(1-a) \in(1-a) A$, откуда $a(1-\varphi(a))=a \varphi(1-a)=0$. Тогда $a=a \varphi(a)+a(1-\varphi(a))=a \varphi(a)=\varphi(a)$.

(3) По лемме 2.8(1) конечномерное слева кольцо $A$ прямо-конечно. В силу (2) $A$ полупримитивно, каждый его регулярньй слева элемент обратим и $\varphi(e)=e$ для каждого центрального идемпотента $e \in A$. Полупервичное правое или левое кольцо Голди $A$ является правым или левым порядком в полупростом артиновом кольце $Q$. Так как все регулярные элементы кольца $A$ обратимы, то $A=Q$.

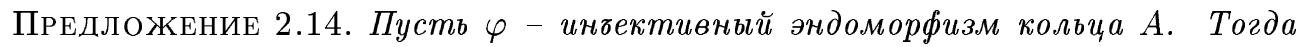
равносильны следуюшие условия:

(1) $A_{r}[x, \varphi]-$ кольио главных левых идеалов;

(2) $A$ - левое или правое кольцо Голди, $A_{r}[x, \varphi]-$ конечномерное слева кольцо и $A_{r}[x, \varphi] / x^{2} A_{r}[x, \varphi]$ - левое кольио Безу;

(3) $A$ - артиново полупростое кольио, $\varphi$-автоморфизм и $\varphi(e)=е$ для каждого иентрального идемпотента е $\in A$.

ДокАЗАТЕЛЬСТво. Импликация $(1) \Longrightarrow(2)$ проверяется непосредственно. Импликация $(2) \Longrightarrow(3)$ следует из лемм $2.13(3)$ и $1.8(8)$.

$(3) \Longrightarrow(1)$. Так как $\varphi$ - автоморфизм, то $A_{r}[x, \varphi]$ совпадает с левым кольцом косых многочленов $A_{\ell}\left[x, \varphi^{-1}\right]$. В кольце $A_{\ell}\left[x, \varphi^{-1}\right]$ коэффициенты стоят слева от $x$ и $x a=$ $\varphi^{-1}(a) x$ для всех $a \in A$. Если $e$ - центральньй идемпотент кольца $A$, то $\varphi(e)=e=$ $\varphi^{-1}(e)$. По левостороннем аналогу предложения $2.12 A_{\ell}\left[x, \varphi^{-1}\right]$ - кольцо главных левых идеалов.

\section{СПИСОК ЦИТИРОВАННОЙ ЛИТЕРАТУРЫ}

[1] Tuganbaev A. A. Semidistributive Modules and Rings. Dordrecht-Boston-London: Kluwer Acad. Publ., 1998.

[2] Hirano Y., Hong C.-Y., Kim J.-Y., Park J.K. On strongly bounded rings and duo rings // Comm. Algebra. 1995. V. 23. №6. P. 2199-2214.

[3] Tuganbaev A. A. Distributive Modules and Related Topics. Amsterdam: Gordon and Breach Sci. Publ., 1999.

[4] Jategaonkar A. V. Left principal ideal rings // Lecture Notes in Math. 1970. V. 123. P. 1-145.

[5] Фейс К. Алгебра: кольца, модули и категории. Т. 2. М.: Мир, 1979.

[6] Armendariz E. P., Fisher J. W. Regular P.I.-rings // Proc. Amer. Math. Soc. 1973. V. 39. №2. P. 247-251.

[7] Michler G.O., Villamayor O.E. On rings whose simple modules are injective // J. Algebra. 1973. V. 25. P. 185-201.

Московский энергетический институт 